\title{
Novel endoscopic ligation with 0 -ring closure involving muscle layer of a gastric artificial defect
}

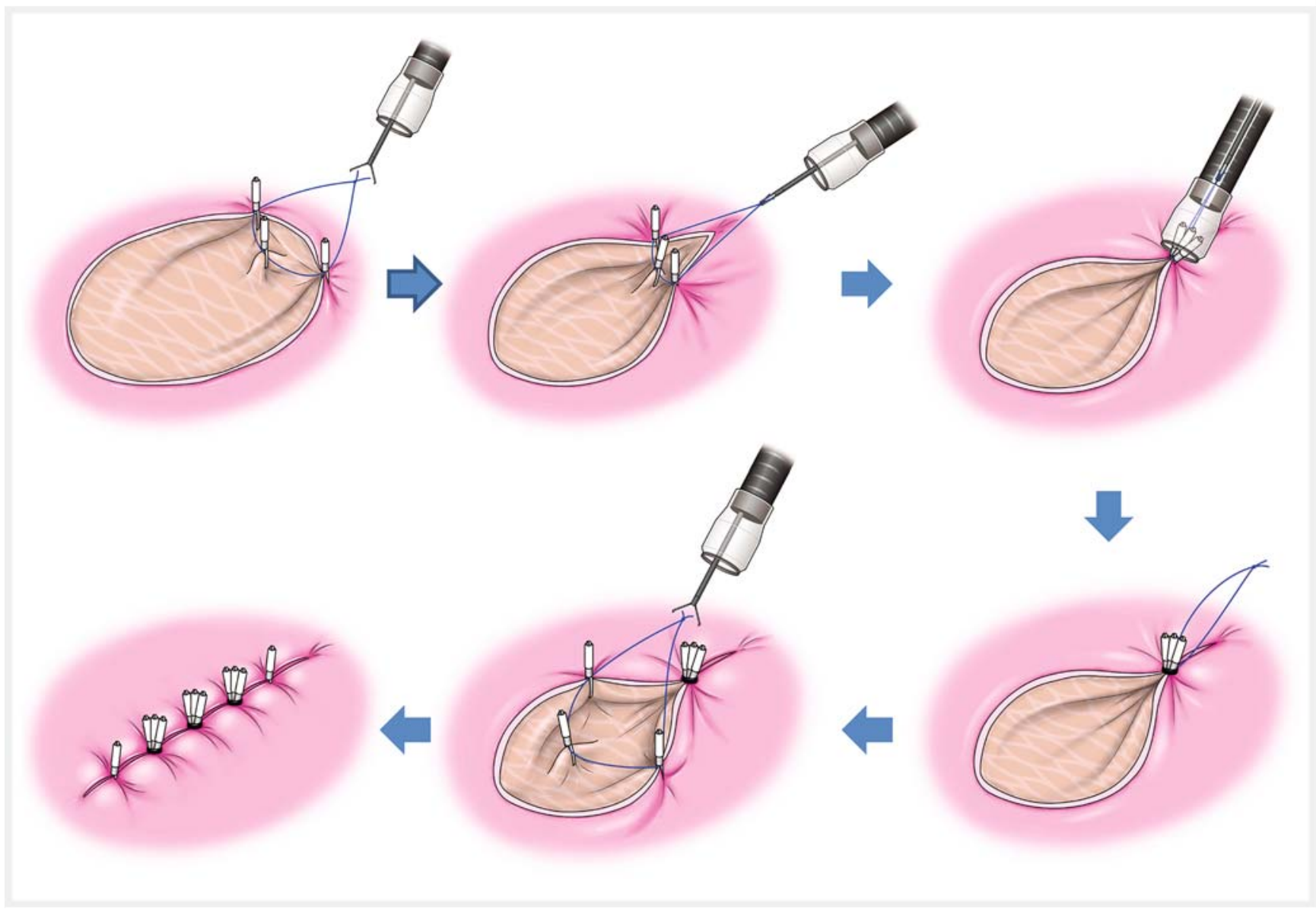

- Fig. 1 Schema showing the endoscopic ligation with O-ring closure (E-LOC) procedure. Source: Davinci Medical Illustration Office.

Endoscopic closure of a gastrointestinal artificial defect effectively reduces the incidence of postoperative adverse events [1], especially delayed bleeding due to antithrombotic drugs. However, although several closure techniques have been developed, including endoloop [2], multiple clip-and-line [3], and hand suturing [4], there is still no convenient and reliable closure technique for the stomach because of the thick gastric wall. One important issue is that limited mucosal suturing results in the formation of a mucosal bridge and cavity, leading to mucosal dehiscence at an early stage. We describe a novel gastric artificial defect closure method named endoscopic ligation with O-ring closure (E-LOC).

An 84-year-old man presented with early gastric cancer in the lower stomach. After standard endoscopic submucosal dissection, a defect $40 \mathrm{~mm}$ in diameter remained.

After written informed consent, the defect was closed using the following method involving the muscle layer ( $\triangleright$ Fig. 1, - Video 1). A 3-0 surgical nylon loop, 2 $\mathrm{cm}$ in diameter, was positioned around the defect, and two hemoclips (HX-610090; Olympus, Tokyo, Japan) were used to anchor the loop on both edges of the defect ( $>$ Fig. 2 ), and/or another one was

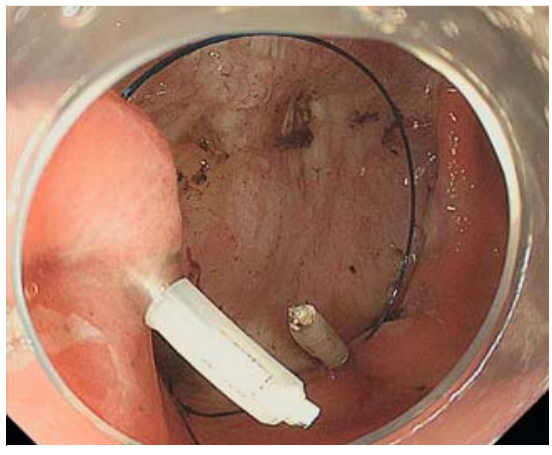

- Fig. 2 A 3-0 surgical nylon loop, $2 \mathrm{~cm}$ in diameter, was positioned around the defect, and two hemoclips were used to anchor the loop on both edges of the defect. 


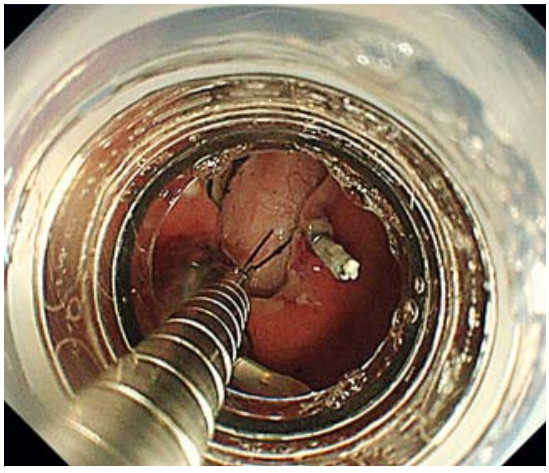

Fig. 3 A clip applicator was used to grasp the loop and pull it into the cap of the endoscopic variceal ligation device.

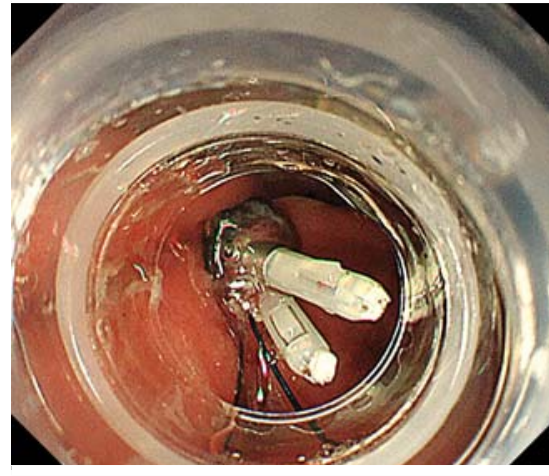

Fig. 4 An O-ring was fired around the hemoclips to close the proximal side of the defect.

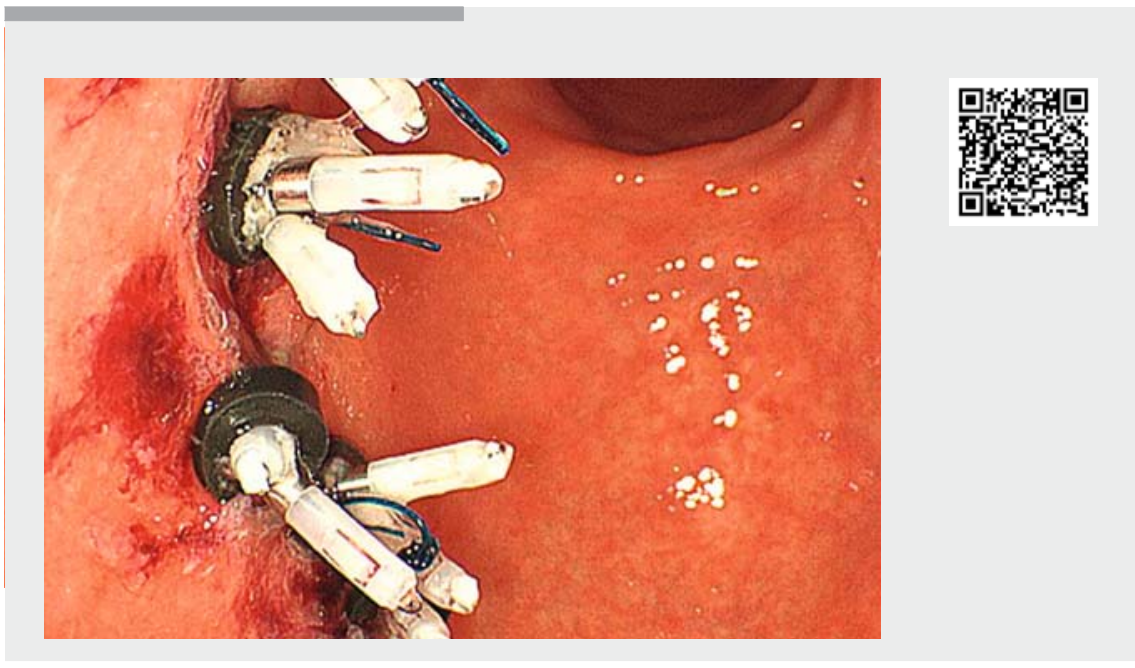

$\checkmark$ Video 1 Procedure used to close the entire gastric artificial defect using an endoscopic variceal ligation device. Source for graphical illustration: Davinci Medical Illustration Office.

applied to the muscle layer. A clip applicator (ZP-S-195S; XMEX, Tokyo, Japan) was then used to grasp the loop and pull it into the cap of an endoscopic variceal ligation device (MD-48720U; Sumius, Tokyo, Japan) ( $\triangleright$ Fig. 3 ); the deployed hemoclips were then pulled into the cap. An $\mathrm{O}$-ring was fired around the hemoclips to close the proximal side of the defect ( Fig.4). This procedure was repeated toward the distal side. Finally, the whole defect was closed completely ( $\mathbf{F i g} . \mathbf{5}$ ). The procedure time was 20 minutes. Three days later, the closure remained intact. This novel method using O-rings and capture of the muscle layer can be a feasible option for closure of large gastric artificial defects.
Endoscopy_UCTN_Code_TTT_1AO_2AN

\section{Competing interests}

The authors declare that they have no conflict of interest.

\section{The authors}

Noriko Nishiyama, Hideki Kobara, Nobuya Kobayashi, Taiga Chiyo, Shintaro Fujihara, Tatsuo Yachida, Tsutomu Masaki Department of Gastroenterology and Neurology, Faculty of Medicine, Kagawa University, Kagawa, Japan

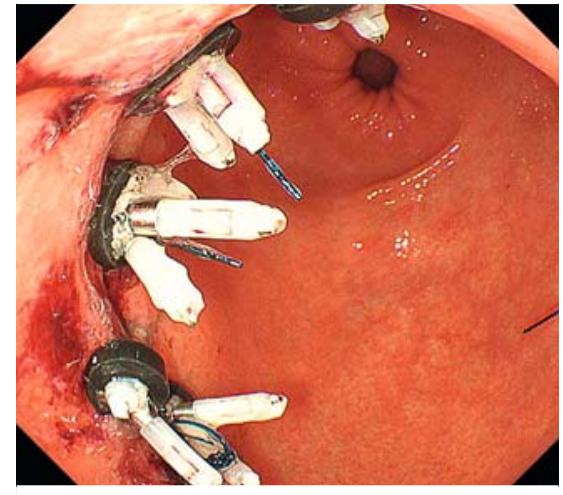

- Fig. 5 Finally, the whole defect was closed completely without a mucosal bridge.

\section{Corresponding author}

\section{Noriko Nishiyama, MD, PhD}

Department of Gastroenterology and Neurology, Faculty of Medicine, Kagawa University, 1750-1 Ikenobe, Miki, Kita, Kagawa 761-0793, Japan Fax: +81-87-8912158

n-nori@med.kagawa-u.ac.jp

\section{References}

[1] Lua GW, Liu F. Closure of a large mucosal defect after endoscopic submucosal dissection using "pre-detached loop and clips" method with a single-channel gastroscope. Endoscopy 2015; 47: E464-E465

[2] Nomura T, Kamei A, Sugimoto $S$ et al. New modified hook device for endoloop closure of the mucosal defect after gastric endoscopic submucosal dissection. Endoscopy 2018; 50: E222-E223

[3] Yamasaki Y, Takeuchi Y, Kato M et al. Lineassisted complete closure of large gastric mucosal defects by use of multiple clip-andline technique. VideoGIE 2016; 1: 49-50

[4] Goto O, Sasaki M, Akimoto T et al. Endoscopic hand-suturing for defect closure after gastric endoscopic submucosal dissection: a pilot study in animals and in humans. Endoscopy 2018; 50: E342-E343

\section{Bibliography}

Endoscopy 2020; 52: E413-E414

DOI 10.1055/a-1149-8597

ISSN 0013-726X

published online 24.4.2020

(c) 2020. Thieme. All rights reserved.

Georg Thieme Verlag KG, Rüdigerstraße 14, 70469 Stuttgart, Germany 